\title{
Secondary Network Throughput Analysis Applying SFR in OFDMA-CR Networks
}

\author{
Joydev Ghosh ${ }^{1 *}$, Varsha Panjwani ${ }^{2}$, Sanjay Dhar Roy ${ }^{2}$ \\ ${ }^{1 *}$ The New Horizons Institute of Technology, Durgapur-8, West Bengal, INDIA,Email \\ \{joydev.ghosh.ece@gmail.com\} \\ 2 National Institute of Technology, Durgapur-9,West Bengal,INDIA,Email \\ \{varsha.panjwani2012@gmail.com,s_dharroy@yahoo.com\}
}

\begin{abstract}
In OFDMA femtocell networks, the licensed spectrum of the macro users (MUs) are available to the femto users (FUs), on the condition that they do not spark off notable interference to the MUs. We contemplate wireless data for femto user (FU) / secondary user (SU) in cognitive radio (CR) networks where the frame structure split up into sensing and data transmission slots. Moreover, we consider soft frequency reuse (SFR) technique to improve secondary network throughput by increasing the macrocell edge user power control factor. SFR applies a frequency reuse factor (FRF) of 1 to the terminal located at the cell centre for that all base stations (BSs) share the total spectrum. But for the transmission on each sub-carrier the BSs are confined to a certain power level. However, more than 1 FRF uses for the terminals near to the macrocell edge area. In this context, we conceptualize the cognitive femtocell in the uplink in which the femtocell access point (FAP) initially perceive by sensing to find out the availability of MU after that FAP revamps its action correspondingly. Appropriately, when the MU is sensed to be non-existent, the FU transmits at maximum power. In other respect, the FAP make the best use of the transmit power of the FU to optimize the secondary network throughput concern to outage limitation of the MU. Finally, effectiveness of the scheme is verified by the extensive matlab simulation.
\end{abstract}

Keywords: Spectrum Sensing, Signal to Interference Plus Noise Ratio (SINR), Average Power Consumption, Secondary Network Throughput.

\section{Introduction}

Radio frequency spectrums are treated as one of the substantial limited resources in wireless communications which should be used effectively. With such stimulation to decrease power consumption and the reuse of radio resources, there is an inescapable shift towards the deployment of femto cell networks by disintegrating traditional single-cell, single-layer network into multi-layer networks with large secondary network throughput [12]. Femtocells are low power invention that 
comes up with better coverage to portable users through femtocell access point (FAP) at the indoor scenario. The access node named as FAP, perform as the base station (BS) for the femtocell and take help of internet as backhaul to get connected with MBS. But, it is crucial to emerge effective interference management scheme in order to make sure the coexistence of the two-tier networks [1], [2]. In [3], SFR technique is introduced to keep away from the inter-tier interference between primary and secondary networks [16]. This is obtained by partitioning the total accessible band into a number of sub-bands and put a limit on the femtocell network's access.
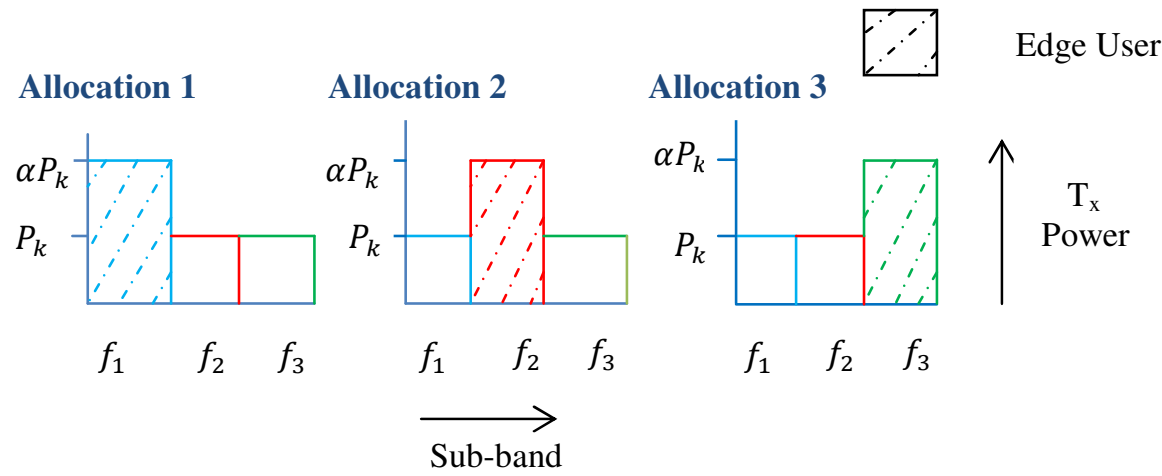

Fig.1 SFR technique in intra-tier: Sub-bands and transmit power allocations at cell edge area for $\mathrm{FRF}=3$.

In a CR networks, the SUs are permitted to use the spectrum of PUs when these frequency bands are not been under utilization. To execute this frequency reuse mechanism, the SUs are required to sense the radio frequency scenario, and once the PUs are found to be active, the SUs are needed to evacuate the channel within a certain period of time. Thus, spectrum sensing is of notable importance in CR networks. The variables connected with spectrum sensing are probability of detection $\left(P_{d}\right)$ and probability of false alarm $\left(P_{f}\right)$ [4]. When high the $P_{d}$, the PUs are more protected from harmful interference. Moreover, from the SUs point of view, lower the $P_{f}$, more chances that the channel can be reutilized when it is accessible, thus larger the secondary network throughput. In this paper, we review the issue of sensing period to optimize the secondary network throughput under the limitation that PUs are sufficiently protected [17]. Consequently, a typical frame structure is contemplated for the SU which composed of the sensing and the data transmission periods [5]. The sensing period and the data transmission period are needed to be incorporated in a unit frame such that (i) the quantity of transmitted data packets become greater and (ii) the number of clashes with the PUs become less. 
In Particular, the major contributions of this paper are highlighted below-

- In order to construct the sensing-throughput trade-off issue, the objective turns out to be reducing, $P_{f}$, under the limitation of $P_{d}$. We therefore construct the sensing-throughput trade-off issue from this viewpoint.

- As macrocell edge users' compromise the spectrum with adjacent macrocells, their uplink transmission adapts higher power level to combat cross tier interference. For the adaptation of above power control mechanism, we consider $P_{\text {centre }}=P_{k}$ and $P_{\text {edge }}=\alpha P_{k}$, keeping $\alpha \geq 1$.

- The performance metric of interest, we refer as secondary networkthroughput, is a complete unit of data that is successfully transmitted in a particular amount of time.

The remainder of this paper is organized as follows. In Section 2, we describe system model to define propose network. In Section 3, the assumptions considered for execution of exact scenario in the simulation model. In Section 4, we present and analyse the numerical results. Ultimately, we finish this work in Section 5 with conclusion.

\section{System Model}

We consider a scenario where femtocells are deployed over the existing macrocell network and share the same frequency spectrum with macrocell. Here we focus only on downlink scenario. The downlink communications in a network with one macrocell and $\mathrm{N}_{\mathrm{F}}=4$ number of femtocells is as shown in Fig.1 in which 4 femtocells are located at $(2,2),(-2,2),(-2,-2),(2,-2)$ within the macrocell coverage. In a macrocell, total $\mathrm{N}_{\mathrm{Mug}}$ user equipments (UEs) are randomly distributed within its coverage area.

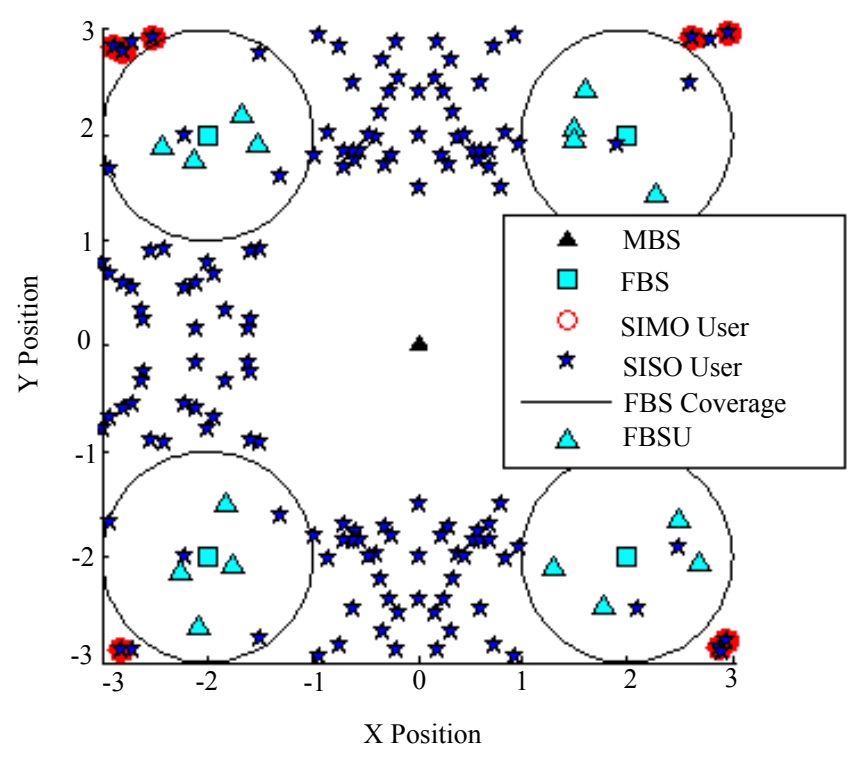


Fig.2 Co-existance scenario of femtocell and macrocell in our proposed network.

In theoretical analysis, we assume that the OFDMA based dual tier network consists of $N_{M}$ number of hexagonal gride macrocell and $N_{F}$ number of femto cells in each macrocell. Total bandwidth associated with the macrocell edge regions is split up into 3 sub-bands by applying SFR technique [6], [7]. A sub-band containing $N_{s c}$ number of sub-channels that are available to provide service to the users located at the cell-centre area and the corresponding cell edge area [14], [15]. Besides, we also consider that the channel is slowly time varying and follows the Rayleigh multipath fading distribution. Three kinds of possible links in duallayer networks are as follows: MBS to outdoor user's link, FBS to indoor user's link, MBS to indoor user's link. Hence, link gain in dual-layer networks can be described as [1]-

$$
G_{n, k, i}^{x}=d^{-\alpha_{p}} 10^{\xi_{s} / 10}|h|^{2}
$$

where $\mathrm{d}_{\mathrm{s} 2 \mathrm{~s}, \mathrm{ji}}$ is the distance between $\mathrm{j}$-th FBS and $\mathrm{i}$-th FBSU. $\xi_{\mathrm{s}}$ (in dB) is a Gaussian random parameter with 0 mean and $\sigma^{2}$, variance, due to shadowing in the channel. Here $|h|^{2}$ denote the channel gain between k-th MBS and its associated ith FBSU. Moreover, Rayleigh fading gives tractable results which assists understanding of the system response to a particular situation. We use the notation $x$ to denote the serving network entity for a generic user. That is, $x=\mathrm{a}$ if the user is associated to a FAP and $x=b$ if the user is associated to a MBS. Without any loss of generic laws, the analysis is conducted on a typical user located at the origin. Therefore, SINR, $\gamma_{n, k, i}^{x}$ at the typical user located at the origin (which also holds for any generic user) served by an MBS or FAP (MBS/FAP) is given by [2]-

$$
\gamma_{n, k, i}^{x}=\frac{P_{n, k, i}^{x} G_{n, k, i}^{x}}{I_{n, k, i}^{x *}+I_{n, k, i}^{\prime x *}+\sigma_{n, k, i}^{2}}
$$

where $G_{n, k, i}^{x}$ is the wireless link gain between the user to the serving network entity (i.e. an MBS or a FAP) over the $n$-th sub-channel. Here $P_{n, k, i}^{x}$ is designated as the proportion of total transmit power by an associated serving network entity over the particular sub-channel. Likewise, the channel gains from a generic location $x \in \mathbb{D}^{2}$ to the MBS, $b_{i}$ and the FAP, $a_{i}$ are denoted by $h_{b_{i}} \sim \sqrt{X_{b_{i}}^{2}+Y_{b_{i}}^{2}}$ and $h_{a_{i}} \sim \sqrt{X_{a_{i}}^{2}+Y_{a_{i}}^{2}}$, respectively, where $X_{x}, Y_{x}$ are indicated as independent gaussian random variables with zero mean and desired variance, $\sigma_{n, k, i}{ }^{2}$ is the noise power of zero-mean complex valued additive white Gaussian noise (AWGN). 
The energy measuring device is composed of a square law device succeeded with an integrator for finite time. The outcome of the integrator at any instant of $t$ is the energy of the input signal to the square law device on a particular interval $(0, T)$. The detection is a measure of the following two hypotheses-

$\mathcal{H}_{o}$ : The input $\mathrm{c}(\mathrm{t})$ is noise alone-

a) $c(t)=n(t) ; n(t)$ denote zero-mean AWGN with unit variance: $n(t) \cong \sigma(0,1)$

b) $E[n(t)\rfloor=0$; where $t$ denotes sample index

c) noise spectral density $=\mathrm{N}_{02}$ (two sided)

d) noise bandwidth equals to $\mathrm{w}$ cycles per seconds

$\mathcal{H}_{1}$ : The input $\mathrm{c}(\mathrm{t})$ is signal + noise -

a) $c(t)=s(t)+n(t)$

b) $\mathrm{E}[s(t) \mathrm{h}(\mathrm{t})+n(t)]=\mathrm{s}(\mathrm{t})$

The tenancy of n-th sub-band is possible to detect with the help of a simple hypothesis test written as [7]

$$
V^{\prime}= \begin{cases}N_{n} ; & \mathcal{H}_{o, n} \\ S_{n}+N_{n} ; & \mathcal{H}_{1, n}\end{cases}
$$

where $n=\left\{1,2, \ldots N_{s c}\right\}, S_{n}$ and $N_{n}$ indicate the descrete frequency response of $s(t)$ and $n(t)$, respectively.

After proper filtering, sampling, squaring and integration, the test statistic of an energy detector is

$$
T_{v}=\sum_{i=1}^{2 r}\left|V^{\prime}\right|^{2}
$$

where $r$ is the number of complex signal samples. As described in [3] the probability density function (PDF) of $T_{v}$ follows a central chi-square distribution with $2 r$ degrees of freedom $(\mathrm{DoF})$ under $\mathcal{H}_{o, n}$, or a noncentral chi-square distribution with $2 r$ DoF and a noncentrality parameter $2 \gamma$ under $\mathcal{H}_{1, n}$. The test statistic, $T_{v}$, is compared with a predefined threshold value $\lambda$. Hence, the probabilities of detection and false alarm can be written as [8], [9]-

$$
P_{d}\left(\gamma_{n, k, i}^{x}, \lambda\right)=Q_{v}\left(\sqrt{2 \gamma_{n, k, i}^{x}}, \sqrt{\lambda}\right)
$$




$$
P_{f}(\lambda)=\frac{\Gamma\left(r, \frac{\lambda}{2}\right)}{\Gamma(r)}
$$

where $Q_{v}(x)=\frac{1}{\sqrt{2 \pi}} \int_{x}^{\infty} \exp \left(-t^{2} / 2\right) d t, \Gamma(a, x)=\int_{x}^{\infty} t^{a-1} \exp (-t) d t, \quad$ and $\Gamma(a, 0)=\Gamma(a)$. Further, missed detection probability can be calculated as $P_{m}\left(\gamma_{n, k, i}^{x}, \lambda\right)=1-P_{d}\left(\gamma_{n, k, i}^{x}, \lambda\right)[10]$.

In general, a frame made up of a sensing period and a transmission period. The sensing period is represented by $\tau$, while total time span of a frame is $T$. In the proposed network model, the sensing time is considered as zero from SUs point of view, as SU not an integral part of spectrum sensing. Additionally, typical value of $\tau$ differs from 0 to $T$ as the introduced network senses the spectrum maximum to the frame duration. The achievable instantaneous data rate of k-th femto user can be calculated by considering two cases, when FAP finds that MU is absent which informs to FU by FAP to transmit at its maximum allowable power $\left(P_{n, k, i}^{\max }\right)$, and when FAP finds that the MU is present for that FAP optimizes $\left(P_{n, k, i}^{*}\right)$ and then, inform femto user to transmit to the FAP. The data rates of FU under the above condition can be written as-

$$
r_{00}=\log _{2}\left(1+\frac{P_{n, k, i}^{\max } G_{n, k, i}^{x}}{\sigma_{n, k, i}}\right)
$$

when FAP accurately senses that the MU not exist.

$$
r_{01}=\log _{2}\left(1+\frac{P_{n, k, i}^{\max } G_{n, k, i}^{x}}{I_{n, k, i}^{x *}+I_{n, k, i}^{\prime x *}+\sigma_{n, k, i}^{2}}\right)
$$

when the FAP inaccurately senses that the MU not exist

$$
r_{11}=\log _{2}\left(1+\frac{P_{n, k, i}^{*} G_{n, k, i}^{x}}{I_{n, k, i}^{x *}+I_{n, k, i}^{\prime *}+\sigma_{n, k, i}{ }^{2}}\right)
$$

when the FAP accurately senses that the MU exists

$$
r_{10}=\log _{2}\left(1+\frac{P_{n, k, i}^{*} G_{n, k, i}^{x}}{\sigma_{n, k, i}{ }^{2}}\right)
$$

when the FAP inaccurately finds that MU exists while MU not exist. 
Hence, secondary network throughput can be given by [5]

$$
T_{f}=E\left\{\left(\frac{T-T_{s}}{T}\right)\left[\begin{array}{c}
r_{00}\left(1-P_{f}(\lambda)\right) P\left(\mathcal{H}_{o, n}\right)+ \\
r_{01}\left(1-P_{d}(\lambda)\right) P\left(\mathcal{H}_{1, n}\right) \\
+r_{11} P_{d}(\lambda) P\left(\mathcal{H}_{1, n}\right)+r_{10} P_{f}(\lambda) P\left(\mathcal{H}_{0, n}\right)
\end{array}\right]\right\}
$$

where $\mathrm{E}\{$.$\} is an expectation operator.$

$$
T_{f}=\left(\frac{T-T_{s}}{T}\right)\left[\begin{array}{c}
R_{00}\left(1-P_{f}(\lambda)\right) P\left(\mathcal{H}_{o, n}\right)+ \\
R_{01}\left(1-P_{d}(\lambda)\right) P\left(\mathcal{H}_{1, n}\right) \\
+R_{11} P_{d}(\lambda) P\left(\mathcal{H}_{1, n}\right)+R_{10} P_{f}(\lambda) P\left(\mathcal{H}_{0, n}\right)
\end{array}\right]
$$

where $R_{00}=E\left\{r_{00}\right\}, R_{01}=E\left\{r_{01}\right\}, R_{11}=E\left\{r_{11}\right\}, R_{10}=E\left\{r_{10}\right\}$.

\section{Simulation Model}

For better approximation of numerical results, Rayleigh fading is included with pathloss and shadowing. The simulation testbed model is carried out considering the following steps-

1. A fixed number of outdoor users' $\left(\mathrm{N}_{\mathrm{MUE}, \mathrm{ku}}\right)$ and indoor users' $\left(\mathrm{N}_{\mathrm{FUE}, \mathrm{ji}}\right)$ is generated and they are randomly distributed within their own coverage area. $\mathrm{N}_{\mathrm{UE}}$ includes all MBSUs /PUs $\left(\mathrm{N}_{\mathrm{MUE}, \mathrm{ku}}\right)$ and FBSUs/SUs $\left(\mathrm{N}_{\mathrm{FUE}, \mathrm{ji}}\right)$ which means $\mathrm{N}_{\mathrm{UE}}$ $=\mathrm{N}_{\mathrm{MUE}, \mathrm{ku}}+\mathrm{N}_{\mathrm{FUE}, \mathrm{ji}}$. Here, $j \in \mathrm{N}_{\mathrm{F}}=\left\{1,2, \ldots \ldots . ., \mathrm{N}_{\mathrm{F}}\right\} ; k \in \mathrm{N}_{\mathrm{M}}=\left\{1,2, \ldots \ldots ., \mathrm{N}_{\mathrm{M}}\right\} ; \mathrm{N}_{\mathrm{MUE}, \mathrm{ku}}$ $\forall\left\{1,2,3, \ldots \ldots, \mathrm{uN} \mathrm{N}_{\mathrm{M}}\right\}, \mathrm{u} \in$ any large integer value; $\mathrm{N}_{\mathrm{FUE}, \mathrm{ji}} \forall\left\{1,2,3, \ldots, \mathrm{i} \mathrm{N}_{\mathrm{F}}\right\}, \mathrm{i} \in$ any large integer value .

2. The interference on k-th user over the n-th sub-channel are executed as below [10],[12],[13]-

$$
\begin{aligned}
I_{k, i}^{f} & =\sum_{\mathrm{l}=1}^{\mathrm{N}_{\mathrm{M}}} P_{i, l, n}^{m} G_{i, l, n}^{m} \forall l \in\left\{1,2,3 \ldots \ldots \mathrm{N}_{\mathrm{M}}\right\} \\
I_{k, i}^{\prime f} & =\sum_{\mathrm{j}=1, \mathrm{j} \neq \mathrm{i}}^{\mathrm{N}_{\mathrm{M} \mathrm{N}_{\mathrm{F}}}} \beta_{j}^{n} P_{i, j, n}^{f} G_{i, j, \not h}^{f} \in\left\{1,2,3 \ldots \ldots \mathrm{N}_{\mathrm{F}}\right\} \\
I_{k, i}^{m} & =\sum_{\mathrm{l}=1, \mathrm{l} \neq \mathrm{i}}^{\mathrm{N}_{\mathrm{M}}} P_{i, l, n}^{m} G_{i, l, \not l l}^{m} l \in\left\{1,2,3 \ldots \ldots \mathrm{N}_{\mathrm{M}}\right\}
\end{aligned}
$$




$$
I_{k, i}^{\prime m}=\sum_{\mathrm{j}=1}^{\mathrm{N}_{\mathrm{M}} \mathrm{xN}_{\mathrm{F}}} \beta_{j}^{n} P_{i, j, n}^{f} G_{i, j, j}^{f}, \forall \in\left\{1,2,3 \ldots \ldots \mathrm{N}_{\mathrm{F}}\right\}
$$

where $P_{i, l, n}^{m}$ and $P_{i, j, n}^{f}$ indicate the transmit signal powers over the $n$-th sub-channel of MBS $l$ and FBS j, respectively; $G_{i, l, n}^{m}$ and $G_{i, j, n}^{f}$ indicate the corresponding path gains for MBS $l$ and FBS $\mathrm{j}$, respectively; $\beta_{j}^{n}$ use as a indicator function for femtocell resource allocation. If $\beta_{j}^{n}=1$ indicates n-th channel is assigned to femtocell $\mathrm{j}$; otherwise $\beta_{j}^{n}=0$.

3. The received signal strength (RSS) is evaluated from PU/MBSU or SU/FBSU at the reference MBS or FBS.

4. Next, the SINR for a PU/Macro user and/or a SU/Femto user are computed.

\section{Results and discussions}

In order to vindicate the excellence predicted by the analytical framework discussed in Section 2, we present several applications at different conditions.

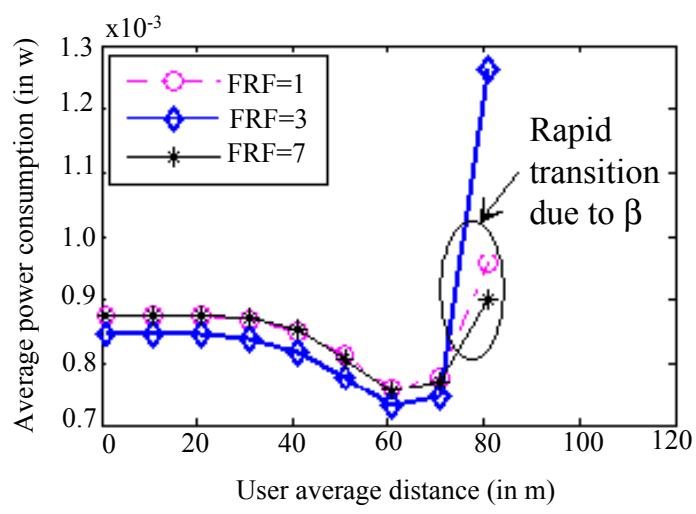

Fig. 3 Average power consumption vs. user average distance for different FRF where number of sub-carriers $(M)=1200$ and sub-carrier spacing $=15 \mathrm{KHz}$

In Fig.3, average power consumption are shown as a function of user average distance for $F R F=1,3$ and 7. Point to be noted that integer FRF is confined to a set of integer numbers: $\{1,3,4,7, \ldots$.$\} followed by the equation i^{2}+i . j+j^{2} \mid i, j \in N$. By applying $F R F=1$ only the users near to the base station (BS) experience better channel quality whereas the users located far apart suffer from poor radio conditions due to sever inter-cell interference (ICI). To accomplish this problem higher 
FRF employs at macrocell edge area. In general, higher FRF reduces the spectral efficiency of the network, but at the same time it (optimum choice, FRF=3) reduces power consumptions. For SFR technique, the total spectrum is split up into three sub-bands: $f_{1}, f_{2}$, and $f_{3}$ as shown in Fig.1. Macrocell edge user are constrained to take service from one of the sub-bands with large transmit power $\left(P_{\text {edge }}=\alpha P_{k}\right)$, while the centre located users can reuse the whole spectrum with a reduced power $\left(P_{\text {centre }}=P_{k}\right)$. When frequency reuse factor is fixed at $F R F=3$,

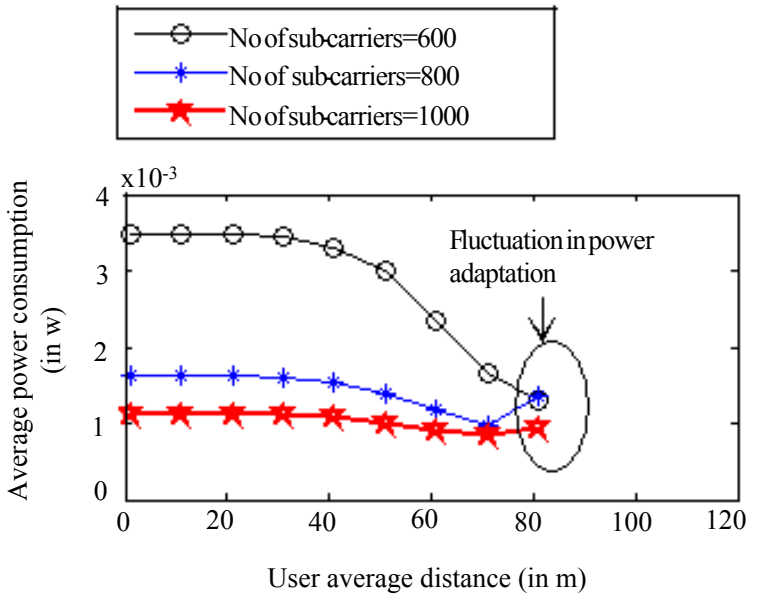

Fig.4 Average power consumption vs. user average distance for different value of $\mathrm{M}$ where $\mathrm{FRF}^{\mathrm{i}}=3$ and sub-carrier spacing $=15 \mathrm{KHz}$

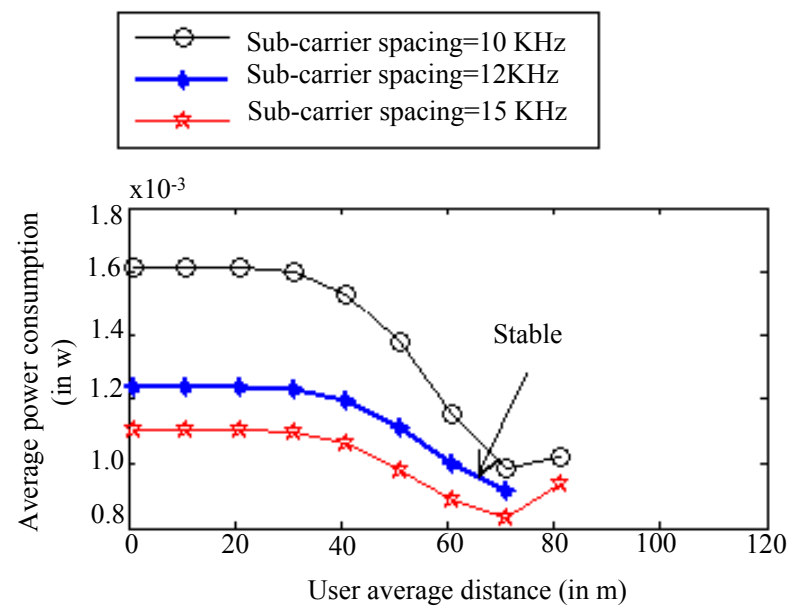


Fig.5 Average power consumption vs. user average distance for different sub-carrier spacing where $F R F=3$ and $M=1200$

the influence of subcarrier spacing and no. of subcarrier parameters on the average power consumption is been investigated in Figs. 5 and 4, respectively. It can be seen that, between subcarrier spacing and no. of subcarrier parameters, subcarrier spacing is more effective factor by means of less power consumption and stability, particularly in the cell edge area.

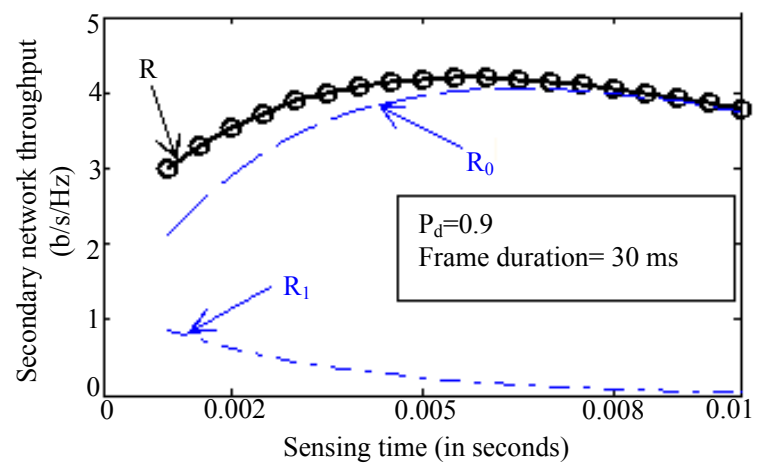

Fig. 6 Secondary network-throughput vs. sensing time where $P_{d}=0.9$ and $T=30 \mathrm{~ms}$

In Fig.6, average network throughput when MUs absent $\left(\mathrm{R}_{0}\right)$ and average network throughput when FUs present $\left(\mathrm{R}_{1}\right)$ are shown as an increasing and decreasing function of sensing time, respectively and the resultant curve $(\mathrm{R})$ is becoming secondary network throughput.

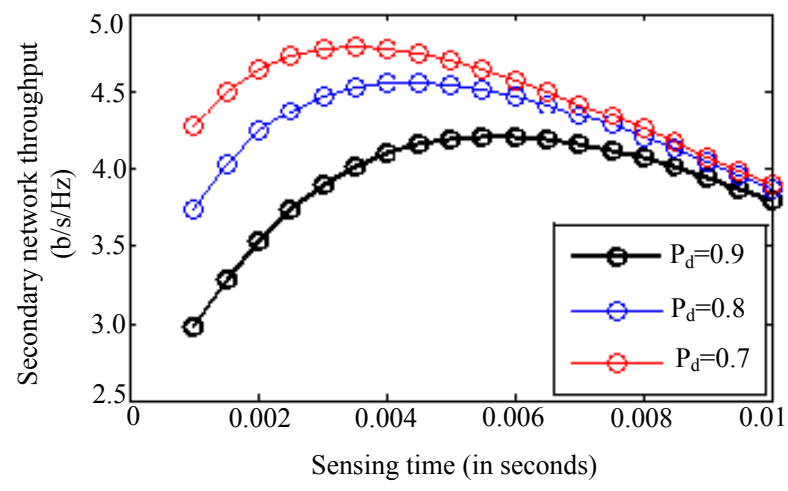


Fig.7 Secondary network throughput vs. sensing time for different $P_{d}$ where $T=30 \mathrm{~ms}$

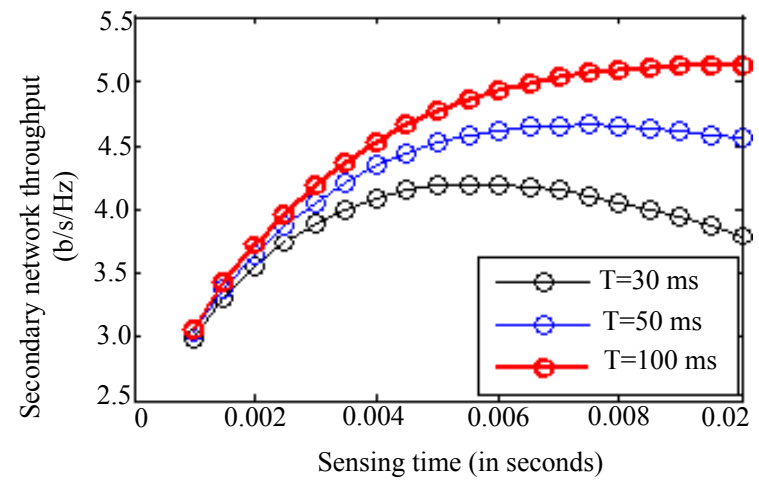

Fig. 8 Secondary network throughput vs. sensing time for different $T$ where $P_{d}=0.9$

Fig.7 plots the secondary network throughput as a function of sensing time for different probability of detection $\left(\mathrm{P}_{\mathrm{d}}\right)$ in imperfect sensing scenarios. However, there is $\left(\frac{T-T_{S}}{T}\right)$ in the secondary network throughput expression in equation no. (12), which can rapidly reduce secondary network throughput when the sensing time is too long. $P_{d}$ is to be set at higher value to make PUs more secure from interference at the cost of secondary network throughput.

Fig. 8 shows the secondary network-throughput when frame duration are $30 \mathrm{~ms}$, $50 \mathrm{~ms}, 100 \mathrm{~ms}$, respectively, and $\mathrm{P}_{\mathrm{d}}$ is set to 0.9 . The issue been raised due to mandatory condition of higher $P_{d}$ and rapidly reduction in secondary network throughput, particularly in the higher sensing time region, can be resolved up to some extend by increasing the frame duration.

\section{Conclusion}

In this paper, we develop a novel simulation testbed model to demonstrate various aspects in terms of substantial parameters in connection with imperfect spectrum sensing and secondary network throughput for dual-tier cognitive femtocell networks. It is been found from the results that we have to compromise with stability to deal with the impact of interference at cell edge area by increasing the power level by the factor of $\alpha$.Tractable analysis of the networks also assist in determining the optimum choice of the parameters which gives insight into the performance tradeoffs of SFR strategies. 


\section{References}

[1] D. Lopez-Perez, I. Guvenc, G. de la Roche, M. Kountouris, T. Q. S.Quek, and J. Zhang, "Enhanced inter-cell interference coordination challenges in heterogeneous networks," IEEE Wireless Commun. Mag., vol. 18, no. 3, pp. 22-30, Jun. 2011.

[2] W. C. Cheung, T. Q. S. Quek, and M. Kountouris, "Throughput optimization in two-tier femtocell networks," IEEE J. Sel. Areas Commun., revised, 2011.

[3] Thomas David Novlan, Radha Krishna Ganti, Arunava Ghosh, Jeffrey G. Andrews, “Analytical Evaluation of Fractional Frequency Reuse for Heterogeneous Cellular Networks," IEEE Transactions on Communications, Vol.60, No.7, July 2012.

[4] Saman Atapattu, Chintha Tellambura, and Hai Jiang,"Analysis of Area under the ROC curve of Energy Detection," IEEE Transactions on Wire-less Communications, Vol.9, No.3, March2010.

[5] ArbabWaheed Ahmad, Heekwon Yang, and Chankil Lee,"'Maximizing Throughput with Wireless Spectrum Sensing Network Assisted Cognitive Radios," International Journal of Distributed Sensor Networks, Hindawi Publishing Corporation.

[6] Thomas David Novlan, Radha Krishna Ganti, Arunava Ghosh, Jeffrey G. Andrews, "Analytical Evaluation of Fractional Frequency Reuse for Heterogeneous Cellular Networks," IEEE Transactions on Communications, Vol.60, No.7, July 2012.

[7] Dong-Chan Oh and Yong-Hwan Lee, "Cognitive Radio Based Resource Allocation in Femto-cell," Journal of Communications and Networks, Vol.14, No.3, June2012.

[8] O. Olabiyi, and A. Annamalai ,"Closed-Form Evaluation of Area under the ROC of Cooperative Relay Based Energy Detection in Cognitive Radio Networks," ICCNC 2012, pp. 1103 - 1107.

[9] Saman Atapattu, Chintha Tellambura, and Hai Jiang,"Analysis of Area under the ROC curve of Energy Detection," IEEE Transactions on Wire-less Communications, Vol.9, No.3, March2010.

[10] Sanjay Dhar Roy, and Sumit Kundu,"Performance Analysis of Cellular CDMA in presence of Beamforming and Soft Handoff," Progress in Electromagnetics Research,PIER,88,pp-73-89, 2008.

[11] J Ghosh, S Dhar Roy," Qualitative analysis for coverage probability and energy efficiency in cognitive-femtocell networks under macrocell infrastructure," ELECTRONICS LETTERS, 20th August 2015, Vol. 51, No. 17, pp. 1378-1380.

[12] Sanjay Dhar Roy, Sumit Kundu, Gianluigi Ferrari , Riccardo Raheli," On spectrum sensing in cognitive radio CDMA networks with beamforming," Physical Communication (2013) Vol.9, pp.73-87.

[13] Gang Ning, Qinghai Yang, Kyung Sup Kwak, Lajos Hanzo,"Macro and Femtocell Interference Mitigation in OFDMA Wireless Systems," Globecom 2012-Wireless Communications Symposium, pp-5290-5295.

[14] S. Misra, P. V. Krishna and V. Saritha, "An Efficient Approach for Distributed Channel Alloca tion With Learning Automata-Based Reservation in Cellular Networks", SIMULATION: Transactions of the Modeling and Simulation International, Vol. 88, No. 10, 2012, pp. 1166-1179.

[15] P. V. Krishna, S. Misra, M. S. Obaidat and V. Saritha, "An Efficient Approach for Distributed Dynamic Channel Allocation with Queues for Real-Time and Non-Real-Time Traffic in Cellular Networks", Journal of Systems and Software (Elsevier), Vol. 82, No. 6, 2009, pp. 1112-1124.

[16] Li Li, Changqing Xu, and Meixia Tao,” Resource Allocation in Open Access OFDMA Femtocell Networks", IEEE Wireless Communications Letters, Vol. 1, No. 6, December 2012.

[17] Zhikun Xu, Geoffrey Ye Li, Chenyang Yang and Xiaolong Zhu," Throughput and Optimal Threshold for FFR Schemes in OFDMA Cellular Networks", IEEE Transactions On Wireless Communications, Vol. 11, No. 8, August 2012. 